\title{
La construcción de la imagen de la Gran China y el discurso de la cooperación internacional: el caso del Foro sobre Cooperación China-África
}

DOI: 10.32870/mycp.v1i1.385

\begin{abstract}
Daniel Ricardo Lemus Delgado
Bajo la guía de la bandera de la paz, el desarrollo y la cooperación, la diplomacia china ha abierto nuevos horizontes y ha avanzado con pujanza sirviendo a la construcción nacional y contribuyendo al mantenimiento de la paz mundial y la promoción del desarrollo común.

Li Zhaoxing, ministro de Relaciones Exteriores de la RPC, 2003-2007.
\end{abstract}

\section{Resumen}

Este artículo tiene como propósito mostrar la relación entre el discurso sobre la cooperación internacional y la construcción de la imagen de la Gran China. Para ello se analiza el discurso de la élite burocrática de la República Popular China en el marco del Foro sobre Cooperación China-África. Este artículo parte del constructivismo como enfoque teórico con el fin de comprender la manera en que las identidades construidas socialmente y las acciones que los Estados asumen condicionadas en parte por dichas identidades, interactúan mutuamente y moldean el escenario internacional. Así, este artículo sugiere que

Artículo recibido el 03 de octubre de 2011 y dictaminado el 24 de enero de 2012.

Daniel Ricardo Lemus Delgado: Profesor-investigador del Departamento de Comunicación y Relaciones Internacionales del Instituto Tecnológico y de Estudios Superiores de Monterrey, Campus Guadalajara. ORCID http://orcid.org/0000-0003-1002-5319 
además de las estructuras materiales que sitúan a China como una potencia en ascenso, este país construye al mismo tiempo la imagen de un Estado que corresponda con este creciente poderío. Así, China construye una identidad en la que se representa como un Estado responsable en la sociedad internacional, que apuesta por un escenario multilateral y que al mismo tiempo se interesa por el desarrollo de los países africanos, desde una óptica distinta. El autor concluye que de esta manera China podría estar generando un modelo alterno de cooperación internacional para el desarrollo que proyecta a este país como una ruta diferente hacia desarrollo en la que los países africanos se pueden apoyar y seguir.

Palabras clave: República Popular China, África, cooperación internacional, constructivismo.

\section{Abstract}

This article illustrates the relationship between the discourse on International Cooperation and the construction of the image of the Great China. Therefore, this article analyzes the discourse of the bureaucratic elite of the Republic of China in the Forum on China-Africa Cooperation. The author assumes a constructivist theoretical approach to understand how socially constructed identities and actions of states determine the international stage. This article suggests that physical structures and identities help to China to build its image in international forums as a rising power. Thus, China is building an identity that is represented as a responsible state in international society, committed to a multilateral setting, based on the principle of nonintervention and equal treatment. Meantime, China is interested in the development of African countries from a different point. The author concludes that China could be developing a different model of international cooperation projects as a different model of development that should be supported and followed for Africans countries.

Keywords: People's Republic of China, Africa, international cooperation, constructivism. 


\section{La construcción de la imagen de la Gran China y la reconfiguración del escenario internacional desde el enfoque teórico constructivista}

El avance que en los últimos 30 años ha experimentado la República Popular China ${ }^{1}$ en el terreno económico, que ha situado a este país como la segunda economía del mundo conforme a su producto interno bruto, ${ }^{2}$ ha sido presentado en los medios de información con una mezcla de incredulidad, asombro y preocupación. En el mundo académico las posturas en torno al impacto que tiene China en el escenario internacional se muestran, en no pocas ocasiones, distantes y contradictorias entre sí. En el fondo estas posturas pretenden determinar de qué manera el escenario internacional puede ser reconfigurado por el factor chino. Para algunos, como Mark Leonard (2008), probablemente estamos en el umbral de una nueva era en la historia mundial, porque el resurgimiento de China tiene repercusiones a mediano y largo plazos que apenas hoy podemos empezar a imaginar y que trastocarán las estructuras del sistema internacional. Desde esta perspectiva, la razón de esta profunda transformación es sencilla: el ascenso de China significa a la vez la reconfiguración del escenario internacional (Gungwu, 2008).

Si bien es cierto que no es posible determinar aún hasta qué punto el ascenso de China representa una posible reconfiguración del escenario internacional, es importante advertir que la percepción de la realidad sobre un mundo en transformación en el que China tiene cada vez mayor injerencia y participación, moldeando lo que el mundo será, ha sido intencionalmente fomentada y acrecentada desde la estrategia comunicativa del gobierno chino, para situar a este país en un lugar distinto al que ocupó en el orden internacional un par de décadas atrás, cuando al triunfo de la Revolución comunista a China se le confirió el rol del simple observador del mundo exterior, mientras que China se encerraba en sí misma en búsqueda de su propio camino hacia el comunismo. ${ }^{3}$

De esta manera, el crecimiento económico ha sido acompañado por una estrategia por parte del Estado para construir la imagen de una "Gran China"

1. De aquí en adelante, China

2. El PIB chino superó los cinco billones 875 mil millones de dólares en el año 2010 (Banco Mundial, 2011).

3. La resolución 2758 de la Asamblea General de las Naciones Unidas, adoptada el 25 de octubre de 1971, reincorporó a China a la ONU después de 22 años de exclusión de este organismo internacional. 
junto a la idea de una "mundo multipolar" (Anguiano, 2008). Esta imagen está siendo construida a partir de diferentes tácticas, que van desde la organización de eventos mediáticos deportivos, hasta la consolidación de un servicio televisivo internacional en inglés por parte de la agencia de noticias estatal Xinhua que refleje el mundo visto desde la visión oficial del gobierno chino ${ }^{4}$ (Lemus, 2010; Xinhua, 2010).

La construcción de la imagen de la Gran China no es un evento fortuito. Evidentemente, la política exterior china es un instrumento privilegiado que el gobierno utiliza para construir y proyectar la imagen de la China del siglo XXI. De esta manera, los cinco principios de coexistencia pacífica de China, como pilar fundamental de la política exterior de este país, no sólo han marcado las pautas del comportamiento del Estado chino, sino también han permitido esbozar la imagen que se tiene sobre lo que debería ser el orden mundial: un sistema multilateral en el que la propia China juegue un rol protagónico (Faust y Kornberg, 1995).

Así, una parte fundamental de la política exterior que va de la mano en el deseo por la construcción de la imagen de esta Gran China es la cooperación internacional para el desarrollo. Si bien es cierto, la cooperación internacional es un instrumento que en ciertas ocasiones permite a un Estado o a un organismo internacional compartir recursos, ideas y estrategias con la intención de apoyar al desarrollo de otros países, sería muy ingenuo suponer que dicha cooperación no persigue a la vez ciertos intereses de los países e instituciones donantes, que van más allá de la simple ayuda para el desarrollo. ${ }^{5}$ China no es la excepción. Su discurso sobre la cooperación, más allá de los mecanismos y estructuras institucionales que permiten concretizar este discurso en

4. La realización de eventos deportivos, más allá de una simple competencia atlética, es un hecho por lo demás bien conocido, ya que éstos representan una oportunidad extraordinaria para generar un discurso propagandístico en el que los logros individuales de los atletas se retoman como triunfos de toda una nación. No es de extrañar entonces el número de estas competencias, que en los últimos cinco años el gobierno chino ha organizado: los Juegos Olímpicos en Beijing en 2008, la Universidad de Invierno en la ciudad de Harbin en 2009, los Juegos Asiáticos en Guangzhou en 2010 y la Universiada de verano en Shenzhen en 2011.

5. Una definición clásica sobre la cooperación internacional es la que propone la Secretaría de Relaciones Exteriores de México, que define la cooperación internacional como "el conjunto de acciones que derivan de los flujos de intercambio que se producen entre sociedades nacionales diferenciadas en la búsqueda de beneficios compartidos en los ámbitos del desarrollo económico y el bienestar social [...] La cooperación internacional así descrita se entiende como la movilización de recursos financieros, humanos, técnicos y tecnológicos para promover el desarrollo internacional" (Secretaría de Relaciones Exteriores, 2010: 1). 
aspectos prácticos, evidencia esta realidad. Particularmente en el caso chino, la cooperación que lleva a cabo es también un medio para construir la idea identitaria - tanto para los chinos como para el resto del mundo- de una China que emerge como potencia mundial y a la vez se preocupa de manera distinta por cooperar con los países en desarrollo.

Para analizar cómo el gobierno chino elabora un discurso que tiene como finalidad proyectar una imagen particular de China respecto a la cooperación para el desarrollo, en este trabajo se aborda el caso de las relaciones del gobierno chino con los países africanos en el marco del Foro sobre Cooperación China-África. Con esta intención parto del supuesto de que el ascenso de China puede ser comprendido como un fenómeno internacional desde el enfoque teórico del constructivismo, porque lo que presenciamos en el caso chino es que tanto las estructuras ideacionales como las materiales se han conjuntado para constituir una nueva figura identitaria de China que explica su participación en el escenario internacional y que a la vez guían sus acciones en el ámbito de la cooperación internacional.

Ahora bien, asumo que aunque los Estados participan en un sistema internacional que está delimitado por estructuras materiales, este sistema puede ser modificado en la medida en que los Estados construyen y se apropian de normas e identidades del propio sistema internacional. Estas normas e identidades son generadas a partir de la postura de las élites burocráticas que construyen y comunican discursos para generar y fortalecer identidades que constituyen, al final de cuentas, un "puente" entre las estructuras y los intereses ideacionales de los Estados, estableciendo una influencia recíproca entre las ideas y aspiraciones y el mundo material que conforma el escenario internacional (Addler, 1997; Santa Cruz, 2009). De esta manera, los Estados pueden moldear el sistema internacional a partir de sus intereses particulares, siempre que sean capaces de construir una identidad colectiva que permita mirarse a sí mismos - $-\mathrm{y}$, de igual forma, ser percibidos por otros agentes internacionales - dentro de las normas que delimitan el campo de acción de sus decisiones en el contexto internacional (Reus-Smit, 2009).

Esta relación dinámica entre las estructuras del sistema internacional y las normas e identidades de los actores está presente, en múltiples niveles, en forma constante en la escena internacional. No es un hecho aislado ni poco común. Tampoco es un hecho que sólo concierna a un grupo reducido de Estados. Al contrario, se trata de actividades cotidianas y constantes a través de las cuales los Estados buscan fortalecer su presencia en el contexto 
internacional. Éste es el caso de China. Así, la construcción y fortalecimiento de una nueva identidad tiene una importancia mayúscula porque permite, al igual que las fuerzas materiales que sostienen su vertiginoso crecimiento económico, moldear la escena internacional con el fin de colocar a China como un país protagonista en el siglo XXI que recién inicia.

Así, el Estado chino está construyendo su imagen de actor internacional como una potencia mundial, vinculando el fortalecimiento del Estado -en un plano meramente material - con una visión idealizada —o al menos incompleta y fragmentada - de la contribución de China al establecimiento de un mundo más pacífico y desarrollado. De esta manera, sugiero que el constructivismo puede ser un marco adecuado para contextualizar la forma en la que el gobierno chino ha encaminado su política exterior en África para sembrar su nueva imagen de lo que China es y quiere ser en un futuro no tan lejano: un país corresponsable en el escenario internacional que impulsa el desarrollo de todos los países sin intervenir en los asuntos internos de cada uno de ellos. Esta construcción de la identidad china va más allá del simple discurso y se materializa en foros y mecanismos específicos, como es el caso del Foro sobre la Cooperación China-África.

Ahora bien, lo que China ha hecho en los últimos 30 años - una combinación de políticas públicas que ha consolidado su crecimiento económico y ha modificado la imagen que proyecta hacia el exterior, mostrándose como un posible socio para el desarrollo que parte de un modelo diferente de cooperación-, puede ser comprendido desde el constructivismo. ${ }^{6}$ Sin embargo, es preciso tener en cuenta, como lo ha planteado Santa Cruz (2009), que el constructivismo más que ser una teoría sustantiva en el campo de las relaciones internacionales, es un marco analítico para estudiar la política mundial. ${ }^{7}$

6. El desafío que plantea China para Occidente, como señala Halper (2010), se encuentra en el hecho de que China ha alcanzado altas y constantes tasas de crecimiento económico en el marco de un Estado autoritario, demoliendo la tesis de que solamente el crecimiento económico constante es posible cuando se combina el liberalismo político con el liberalismo económico. En el caso del Foro sobre Cooperación China-África, una estrategia particular de la cooperación es compartir esta fórmula de un crecimiento económico que no exige la democracia liberal para que éste sea posible.

7. El constructivismo surgió como un intento de adecuar algunos conceptos tradicionales en el campo de las relaciones internacionales para resolver las nuevas inquietudes ontológicas y epistemológicas derivadas del nuevo contexto internacional surgido a partir del fin de la Guerra Fría, cuando ni la visión del neorrealismo ni las otras corrientes teóricas fueron capaces de explicar adecuadamente la vertiginosa transformación del mundo bipolar como consecuencia del fin de la Unión Soviética. 
En este sentido, es preciso recordar que el constructivismo es uno de los nuevos enfoques en el campo teórico de las relaciones internacionales que se ha incorporado vigorosamente en los últimos 20 años y que permite mirar la realidad internacional desde una perspectiva más amplia, que va más allá del debate tradicional entre el realismo y el idealismo (Addler, 1997).

Sin embargo, como marco analítico, los trabajos realizados desde la óptica constructivista difieren unos de otros. Esta diferencia es tan amplia que incluso cuestionan la validez del posible conocimiento obtenido al cuestionar las bases metodológicas y epistemológicas que los sustentan, en posturas que parecen ser inconciliables. El debate está abierto y ha dividido a los pensadores constructivistas entre modernos y posmodernos. ${ }^{8}$ No es el propósito del presente ensayo abonar a este debate, baste con señalar que a pesar de esta diversidad en su conceptualización, el marco analítico es útil en la medida que permite relacionar las estructuras materiales con las ideacionales, señalando posibles pautas de interacción e influencia mutua. El caso chino es evidente en este sentido: en forma dinámica, crecimiento económico, transformaciones materiales, nuevas identidades, eventos mediáticos, política exterior y discurso oficial interactúan en un todo en forma compleja e incansable, generando nuevas interacciones y nuevas formas de comportamiento del Estado. Las pistas para comprender estas interacciones, sugiero, pueden ser proporcionadas desde un enfoque constructivista.

Las diferencias presentes al interior del campo constructivista han generado una idea difusa de lo que comúnmente se interpreta como constructivismo. El punto que se presenta como común es la vaga afirmación de que la realidad social es construida. Sin embargo, es posible partir de dos principios básicos y muy generales de lo que el constructivismo significa: primero, las estructuras que enmarcan la convivencia humana son, ante todo, moldeadas por las ideas comunes, antes que por las fuerzas materiales; y segundo, las identidades y los intereses de los actores son construidos por las ideas comunes, antes que ser dadas por naturaleza (Wendt, 1999). Bajo estas dos premisas, se entiende

8. Existen, en este sentido, diversas clasificaciones sobre las diferentes corrientes al interior del constructivismo, las cuales son categorizadas ya sea por el nivel de análisis que sus investigaciones emprenden, por el fundamento epistemológico en que se basan o por la taxonomía que emplean. La más generalizada de éstas distingue entre un constructivismo positivista y otro pospositivista o posmoderno. En este ensayo las ideas generales del constructivismo y la manera en que se aplican en el análisis de la cooperación hacia África excluyen la postura posmoderna del constructivismo. 
que las instituciones son construidas socialmente a partir de las identidades de los actores, las cuales tienen su origen en una interpretación social de la realidad. ${ }^{9}$ En otras palabras, la realidad social y la forma en que es percibida, cuyos fundamentos evidentemente son materiales, influye en la forma en que los grupos sociales se perciben y perciben a los otros, creando, moldeando o fortaleciendo sus propias identidades. Y lo mismo sucede en el caso de los Estados, que desde las élites burocráticas que los conforman, generan la identidad y el deber ser del Estado en su participación en el escenario internacional.

Desde esta perspectiva, el constructivismo es entendido como el enfoque teórico que propone que la manera en que se forma y se determina el mundo material es resultado de la interacción dinámica entre realidad y acción humana, la cual depende de la interpretación normativa y epistemológica del mundo material (Addler, 1997). ${ }^{10}$ De esta forma, por ejemplo, los grandes discursos de la política exterior de un Estado, acompañados de acciones concretas que los refuerzan, tienen a su vez un impacto en las estructuras materiales del sistema internacional. El punto clave es determinar qué comunican estos discursos, cómo son asociados a las estrategias de la política exterior de los Estados y de qué manera dicha relación entre discurso y política exterior moldea las nuevas identidades de los Estados, tal como está sucediendo actualmente en China.

Ahora bien, a pesar de las diferentes aproximaciones sobre la comprensión del constructivismo como marco teórico, son por lo menos tres los elementos comunes presentes en los diversos trabajos de investigación enmarcados dentro del constructivismo; estos elementos son, conforme a Wendt (2009):

9. Guzzini (2000) ha advertido que es esta idea difusa sobre lo que se entiende por constructivismo lo que ha dado paso a una crítica al interior de los propios constructivistas sobre el uso generalizado de la expresión la "construcción social de...", lo que ha propiciado aproximaciones eclécticas o redundantes en las que incluso se pierde la coherencia teórica en el resultado de las investigaciones. En este artículo parto de postulados básicos aportados por el constructivismo con la intención de que sean referencia para explicar la forma en que el discurso sobre la cooperación internacional enmarcado en el Foro sobre Cooperación ChinaÁfrica, son a su vez ideados, propiciados y enmarcados desde las élites burocráticas para generar y consolidar nuevas identidades del Estado, las cuales fundamentan y dan sentido a la participación de China en el escenario internacional en una lógica que combina tanto las necesidades materiales como las necesidades aspiracionales de dicha élite sobre lo que el futuro de China debe ser.

10. En este sentido, el constructivismo ha sido propuesto como una búsqueda para la reconciliación entre el antiguo debate liberalismo-realismo, al conciliar la visión contradictoria del acontecer internacional, al sostener que las ideas moldean las estructuras del mundo material, como también el mundo material condiciona el surgimiento y consolidación de las ideas (Zhefuss, 2002). 
a) Primero, la afirmación de que las estructuras "normativas" e "ideacionales" son tan importantes como las estructuras materiales. Así como, por ejemplo, las normas institucionales de la academia moldean la identidad de un profesor y, de esta manera, su forma de actuar en contextos específicos a partir de lo que se reconoce como deseable, válido o permitido; las normas del sistema internacional condicionan la identidad social de la soberanía de un Estado y la forma en que éste debe actuar en el escenario internacional.

b) Segundo, la necesidad de comprender de qué manera las estructuras no materiales condicionan las identidades de los actores, debido a que las identidades informan sobre los intereses, y los intereses, a su vez, condicionan las acciones de los actores. Las identidades son las bases de los intereses. En otras palabras, la actuación de un Estado en el sistema internacional depende de aquellos intereses que son, a su vez, configurados por las identidades sobre las que se sostienen. Comprender cómo los Estados desarrollan sus intereses es crucial para explicar el rango de los fenómenos políticos en la arena internacional que un enfoque racionalista ignora o no comprende en forma plena.

c) Tercero, la aseveración de que los Estados y las estructuras son mutuamente constituidos. Las normas institucionalizadas y las ideas definen las características e identidad de los actores individuales y los patrones de las actividades políticas, económicas y culturales realizadas por las personas. Por ejemplo, es a través de la rutina (repetición) de los actos -derechos humanos, libre comercio- como continúan las prácticas del liberalismo. Los mecanismos a través de los cuales se "moldea" la identidad de los actores son la imaginación, la comunicación y la limitación. Es así como las normas e ideas funcionan como racionalizadores de los comportamientos debido a la fuerza moral que se adquiere en un contexto social. Así, más allá de señalar que los actores internacionales son entes egoístas o cooperadores, lo que en realidad los distingue es que son agentes profundamente sociales, respondiendo a los intereses que son creados desde la construcción de las identidades. Los intereses de los actores son endógenos como un producto de reflexión social. De esta manera, se puede afirmar que el campo de las relaciones internacionales es un reino constituido socialmente. 
Si aceptamos estas tres premisas propuestas desde el enfoque constructivista, entonces se puede afirmar que la elaboración y comunicación de discurso son un medio privilegiado para construir y/o fortalecer las identidades colectivas. En el caso chino, analizar la postura oficial del Estado sobre lo que aspira que sea el escenario internacional en cuanto a las relaciones que deben presentarse entre los diferentes Estados, particularmente las relaciones de cooperación, es un elemento más que ayuda a comprender lo que el ascenso de la Gran China significa para el escenario internacional y la forma en que este ascenso puede estar enmarcado ideacionalmente para su concretización.

Evidentemente, la formación de estas identidades colectivas no sucede en un terreno neutral, antes bien, en un proceso dinámico parten de realidades concretas que reflejan los intereses de un grupo particular que guía y orienta la acción del Estado. Es un acto de ingenuidad el pensar que la construcción de identidades es un hecho neutral, objetivo y racional. Al contrario, la experiencia indica que las identidades se construyen y comunican en un reflejo de los intereses de un grupo sobre otros - a veces velado, a veces evidenteque dinamizan la acción social. Y es, en el caso chino, la visión de la élite del Partido Comunista la que intencionalmente se proyecta en los discursos y acciones de la política exterior para conformar la identidad en el escenario internacional de una Gran China. ${ }^{11}$

\section{La cooperación internacional desde la política exterior china como eje para la construcción de una nueva imagen de China}

El discurso sobre la cooperación internacional, particularmente los vínculos que existen entre China y África, ha sido presentado desde el marco de la política exterior china, la cual tiene como eje rector los "cinco principios de coexistencia pacífica”. Estos principios son el respeto mutuo a la soberanía e

11. Una de las críticas que frecuentemente se realizan sobre el constructivismo es que aún no se ha desprendido del todo de una visión estatocéntrica del escenario internacional, presentando en forma velada que los Estados son actores unitarios en el escenario internacional. La realidad, evidentemente, es más compleja que esto. Incluso, en un caso como en China donde el sistema autoritario del gobierno se muestra más cohesionado hacia el exterior, al interior de la cúpula del Partido Comunista existen y han existido diferencias entre las élites. Sin embargo, el mismo caso chino muestra hasta ahora que existe una congruencia en la forma de actuar en el escenario internacional, particularmente en África, como producto de la capacidad de proyectar una imagen de una y única China a pesar de la diversidad étnica, lingüística y cultural que este mismo país representa. 
integridad territorial, la no agresión, la no intervención en asuntos internos, la búsqueda de la igualdad y de beneficios recíprocos, y la coexistencia pacífica. ${ }^{12}$

Bajo los principios de la coexistencia pacífica, en el año 2005 el entonces ministro de Relaciones Exteriores, Li Zhaoxing, presentó a la comunidad internacional un documento titulado La paz, el desarrollo y la cooperación: Bandera de la diplomacia china en la nueva época. En ese documento se establecía que en los albores del siglo xxi el escenario internacional experimentaba profundos cambios derivados de la globalización, la multipolaridad y el avance de la ciencia y la tecnología que exigían adoptar por parte de "todos los pueblos del mundo" la meta común de preservar la paz y promover un desarrollo compartido (Li, 2005).

Desde esta perspectiva, Li afirmaba que la paz era el único camino para el desarrollo de la propia China y que las relaciones internacionales que China mantenía con el resto del mundo estaban enmarcadas por los cinco mil años de historia de esta civilización, las cuales habían sido marcadas por "la cordialidad, la benevolencia y la buena vecindad" en la búsqueda de constituir un mundo en el que fuera posible vivir en armonía. ${ }^{13}$ Este mundo pacífico y armonioso sólo sería posible en la medida en que "todos los países, grandes o pequeños, ricos o pobres y poderosos o débiles, deban tratarse en pie de igualdad y convivir en amistad" (Li, 2005: 2).

De esta manera, la política exterior china se había encaminado, conforme a Li (2005), a favorecer el multilateralismo, la democratización y la legitimización de las relaciones internacionales con la intención de establecer un orden mundial justo y razonable. Al mismo tiempo, la postura oficial de China reflejada por Li proclamaba un nuevo concepto de desarrollo centrado en la

12. Estos principios fueron proclamados por China en el año de 1954. Un año más tarde, en la Conferencia de Bandung, ante la presencia de 29 representantes de Estados africanos y asiáticos, fueron propuestos por el gobierno chino encabezado por el primer ministro Zhou Enlai como base para la cooperación entre los países en vías de desarrollo (CRI, 2005).

13. Es interesante el uso de la historia para la justificación del presente que el gobierno chino utiliza frecuentemente con la idea de proyectar una imagen sobre un pasado de China en que se muestra a este país no solamente como una gran civilización, sino como una civilización que es una y única a la vez y que ahora, la República Popular, es la heredera legítima de esta civilización (Lemus, 2010). Por eso no es de extrañar que este documento haga referencia el pensamiento clásico de China, reflejada en la regla de oro propuesta por Confucio en el siglo VI a. C. en la idea de "no hacer a otros lo que no se quiera que se haga con uno mismo", y en el ideal confucionista de la armonía como elemento fundamental del orden social, en este caso, del orden internacional. 
igualdad y el beneficio mutuo. Y en cuanto al concepto de seguridad, se establecía que debía basarse fundamentalmente en la confianza mutua, el beneficio recíproco, la igualdad y la colaboración, por medio del diálogo y el establecimiento de mecanismos de cooperación para la seguridad regional.

Un aspecto significativo en la visión de Li es la propuesta de hacer valer un nuevo concepto de civilización caracterizado por el respeto a la diversidad. Teniendo como punto de partida la idea de considerar que la diversidad de las civilizaciones mundiales constituye "el patrimonio común de las sociedades humanas y la fuente valiosa para alcanzar la prosperidad", destacaba como un hecho inalienable que cada pueblo escogiera por sí mismo el camino al desarrollo conforme a sus condiciones concretas ( $\mathrm{Li}, 2005$ : 3 ).

El principio de la no interferencia en los asuntos internos de los Estados, sin duda alguna ha resultado significativo para las naciones africanas. Para numerosos países africanos la relación con China constituye un medio que les permite alcanzar financiamiento externo y obtener ayuda para el desarrollo sin necesidad de verse sometidos al escrutinio exigente sobre su gobierno, que por lo general los países occidentales requieren. Y, al mismo tiempo, como señala Halper (2010), el modelo de desarrollo chino se muestra como una experiencia diferente y exportable, en el que se combina una fórmula distinta basada en el crecimiento económico sin la democracia liberal. En otras palabras, en su relación con los gobiernos africanos el gobierno chino se muestra a sí mismo como un socio confiable porque no exige cuentas sobre las decisiones internas del Estado - o no lo hace, al menos, en forma tan 
evidente- - y propone modelos de desarrollo en donde los principios básicos de la democracia liberal pueden quedar a un lado.

En el hilo conductor del discurso de Li la cooperación resulta fundamental porque es un medio para mantener la seguridad internacional. Li establece que "Sólo mediante la cooperación internacional se puede solucionar eficazmente el problema de seguridad común de los países. Son inviables la mentalidad de guerra fría, el unilateralismo y la primacía de la fuerza" (Li, 2005: 10).

Al mismo tiempo, la cooperación que permita realizar el desarrollo común debe basarse en la premisa de que una economía globalizada requiere de una cooperación globalizada. La cooperación es vista además como el camino necesario para impulsar la coexistencia armoniosa entre las distintas civilizaciones porque permite el respeto, el diálogo y la confianza mutua. Y en el caso de la cooperación con los países en vías de desarrollo, Li la describe como un "pilar fundamental de la diplomacia china" (Li, 2005: 11).

En cuanto la postura china respecto a la cooperación Sur-Sur, el Ministerio de Asuntos Exteriores elaboró en el año 2003 un documento guía en el que se expone la conceptualización de este tipo de cooperación desde la postura gubernamental. En ese documento el Ministerio destacaba que los países en vías de desarrollo, que representan $80 \%$ de la población mundial y que cuentan con numerosos recursos naturales y enormes potencialidades de mercado, podrían apoyarse unos con otros para el beneficio mutuo (MAE, 2003). Este beneficio puede partir del hecho de "tomar experiencias ajenas como referencia y procurar un desarrollo común” (MAE, 2003: 1). Evidentemente esta experiencia ajena, que debería servir como modelo de desarrollo, indiscutiblemente es el caso chino:

Como uno de los países en vías de desarrollo, China está dispuesta a desarrollar, en forma amplia y profunda, la cooperación económica, científica y tecnológica, educacional y cultural con los países del Sur sobre la base de igualdad y beneficio mutuo y poniendo énfasis en conseguir resultados prácticos, diversificar las formas y lograr un desarrollo común. A los países subdesarrollados que tienen ciertas dificultades, China está dispuesta a ofrecerles asistencia que está a su alcance. La asistencia china es limitada; sin embargo, refleja su sinceridad, pues no impone ninguna condición" (MAE, 2003: 1).

El planteamiento del Ministerio de Asuntos Exteriores es interesante desde la perspectiva de la conceptualización de la misma China en el escenario internacional, que parte de los siguientes supuestos. En primer lugar, China 
se mira a sí misma como un país en vías de desarrollo, que aunque ha experimentado un crecimiento económico notable en las últimas décadas, éste aún no es suficiente para ubicarse como un país de consumo medio que le permita ser parte del club de países desarrollados. En segundo lugar, China puede resultar ser un socio más empático con aquellos países que también se encuentran en el camino del desarrollo, por lo que sugiere un trato desde una aproximación diferente del escenario internacional; dicha aproximación lleva a la necesidad de cooperar para alcanzar metas en común, desempeñando así el papel del hermano mayor en un mundo cooperativo pero jerarquizado. Y, en tercer lugar, China fomenta un modo diferente de cooperación que no "exige condición", es decir, que no espera pautas de conductas específicas de los Estados para ofrecer su cooperación.

Esta visión de la cooperación y el desarrollo es complementada por el documento sobre el Desarrollo pacífico de China que presentó a la opinión pública la Oficina de Información del Consejo de Estado en septiembre de 2011. En este documento se advierte que el ascenso de China para los demás países significa que: "China no puede desarrollarse en forma aislada del resto del mundo, y la prosperidad y la estabilidad no puede mantenerse sin China" (OICE, 2011: 6). La idea que permea es que los logros de China son inseparables de su cooperación porque el desarrollo de China es imposible sin la comprensión y el apoyo del resto del mundo (OICE, 2011).

Esta misma visión fue expresada por el presidente Hu Jintao en la tercera reunión de los líderes de los BRIC en la ciudad de Sanya, en la provincia de Hainan, realizada en el mes de abril de 2011. En esta reunión Hu declaró que a inicios del siglo XXI, aunque el mundo se ha movido hacia la globalización económica y la multipolaridad, todavía hay "hay un largo camino por recorrer antes de que la paz duradera y prosperidad común se puede lograr" (Hu, 2011: 1). Ante esta encrucijada, Hu planteó que el gran desafío por resolver por parte de la comunidad internacional es cómo encontrar los caminos que lleven a que el siglo XXI sea una época caracterizada por un desarrollo pacífico que permita a la humanidad disfrutar de una prosperidad común.

$\mathrm{Hu}$ planteó cuatro medidas que reflejan la visión discursiva de la élite burocrática china respecto a cómo proceder en el escenario internacional. La primera de estas medidas es mantener la paz y la estabilidad mundial como una condición indispensable para el desarrollo, enmarcado en los propósitos y principios de la Carta de las Naciones Unidas. Una segunda medida apunta hacia la promoción del desarrollo común de todos los países, lo que implica 
apuntalar el crecimiento económico mundial. ${ }^{14}$ Una tercera acción establece el fortalecimiento de los mecanismos de cooperación e intercambio internacional. Finalmente, se advierte la necesidad de fortalecer la asociación de los BRIC para el desarrollo común a partir de los principios básicos de solidaridad, confianza mutua, apertura y transparencia (Hu, 2001).

En su conjunto, la perspectiva sobre el desarrollo y la cooperación que de manera continua ha expresado la élite burocrática china, evidencia que la visión que China propone sobre la cooperación y el desarrollo va más allá del impacto económico, pues, como señala Jaques (2009), la influencia china es más política y cultural en un sentido tan profundo que puede dar origen al nacimiento de un nuevo orden internacional. Es esta visión la que ha delimitado la relación entre los países africanos y China, particularmente el Foro sobre Cooperación China-África.

\section{El discurso chino sobre la cooperación en las relaciones con África}

En la última década, particularmente en los últimos cinco años, los vínculos entre los países africanos y China se han incrementado sustancialmente. China ya se ha convertido en el tercer socio comercial más importante de la región, permeando su influencia a lo largo y ancho del continente. Una sombra china recorre el continente africano, teniendo múltiples impactos para las sociedades africanas. Como lo ha observado Rotberg (2008), China y África se necesitan desesperadamente uno al otro, fortaleciendo así una relación simbiótica.

Estos vínculos económicos y comerciales, pero también políticos y culturales, no son fortuitos, ya que desde una interpretación constructivista, estos lazos se desprenden de la visión que China ha tenido sobre África y la construcción de la propia imagen identitaria que la China contemporánea ha generado de sí misma. De esta manera, la historia de las relaciones diplomáticas que China ha instaurado con los diferentes países africanos y el establecimiento de foros y mecanismos para impulsar una relación económica, política y cultural más estrecha, es parte de un proceso amplio, complejo y dinámico en el que las ideas e identidades se mezclan y funden con las necesidades y

14. Es preciso recordar que desde el año 2000 la contribución china al crecimiento económico mundial del PIB, en términos de poder adquisitivo, ha sido mayor que la de Estados Unidos; representa, asimismo, más de la mitad de la contribución conjunta de India, Rusia y Brasil (Lora, 2005). 
aspiraciones materiales. En otras palabras, tanto el discurso sobre "el deber ser" del escenario internacional que plantea China a través de su élite burocrática, como las necesidades materiales que China requiere -energéticos, mercados, materias primas- moldean las estructuras internacionales, a la vez que condicionan la participación de China en África.

Fundamentalmente, el discurso chino sobre las relaciones internacionales se desprende de una visión basada en el hecho de que tanto los países africanos como China vivieron las consecuencias del imperialismo europeo en sus propios territorios durante el siglo XIX. Cada Estado, en su propia circunstancia histórica, encontró caminos para su liberación - liberación que no todos los Estados han ganado plenamente- (Rupp, 2008). Desde esta experiencia, conforme a este discurso China respeta la soberanía de los Estados africanos y no pretende establecer una hegemonía política o económica sobre África ni ocupar el estatus que antiguamente ocuparon los Estados colonialistas. Por lo tanto, la presencia de China en África se presenta como la imagen de un país cuyo interés por la cooperación es cualitativamente distinto. ${ }^{15}$

Así lo expresó, por ejemplo, Du Xiaconcog, miembro de la Misión Permanente de China ante la ONU en un simposio celebrado en la Universidad de Syracuse, cuando refirió que China y África son "amigos en la adversidad". Considerando el enorme potencial que África representa para el mundo, la cantidad de recursos que posee y el número de personas que lo habitan, Du precisó el rol que a China le gustaría establecer; habló de "una relación constructiva y estratégica de la cooperación para beneficio mutuo y desarrollo común con África y nuestros hermanos y hermanas de África, lo que contribuye a salvaguardar la paz mundial y la prosperidad común" (Du, 2010: 1).

En este discurso Du (2010) recordó los principios básicos que determinan la relación China-África establecidos en el año 2006. Estos principios son:

15. Claro está que existe siempre una distorsión entre el discurso que construye nuestras identidades y las bases materiales que lo soportan. China no es la excepción, ya que como lo advierte la propia Rupp (2008), la interacción de las estructuras materiales de China y de los Estados africanos favorece una relación que se asimila más a las antiguas prácticas de los países colonizadores que un auténtico movimiento solidario y desinteresado. Sin embargo, es importante tener en cuenta que éste es un discurso repetido que ayuda a construir y consolidar una imagen de China en su relación con África; en este sentido, desde el campo de las identidades el discurso sirve para dar sentido y significado a lo que China aspira a ser en el escenario internacional, tanto en la parte identitaria como en la material. 
- La sinceridad, la amistad y la igualdad, teniendo como punto de partida los "Cinco principios de coexistencia pacífica", respetando así la elección soberana que los países africanos hacen sobre el modelo de desarrollo que quieran seguir y apoyando a dichos países en los esfuerzos que realizan para fortalecer su unidad política.

- El beneficio mutuo, la reciprocidad y la prosperidad común. China apoya los esfuerzos de los países africanos para el desarrollo económico, llevando a cabo medidas para la cooperación en diversas formas que impactan en el desarrollo económico y social, y a la vez promueven la prosperidad común.

- El apoyo mutuo y una estrecha coordinación. China busca la cooperación con África en los mecanismos multilaterales como el de las Naciones Unidas, mediante el apoyo a las demandas razonables que proponen los países africanos y apelando a la comunidad internacional para que se preste más atención a las cuestiones relativas a la paz y el desarrollo en África.

- La búsqueda del desarrollo común y el aprendizaje mutuo. China y África deben aprender y aprovechar las experiencias mutuas en materia de gobernanza y de desarrollo, fortaleciendo el intercambio y la cooperación en campos como la educación, la ciencia, la cultura y la salud. China trabajará junto con África en la exploración de la vía del desarrollo sostenible apoyando los esfuerzos de los países africanos para fomentar sus propias capacidades para el desarrollo. ${ }^{16}$

Los principios básicos que enmarcan la cooperación China-África se originan de la visión de la élite burocrática sobre las características del contexto inter-

16. Es más que evidente que estos principios para la relación China-África no podían dejar a un lado la cuestión de Taiwán para el establecimiento de relaciones diplomáticas con aquellos Estados africanos que aún no tenían relaciones diplomáticas con China. "El principio de una sola China constituye la base política para el establecimiento y desarrollo de las relaciones de China con los países africanos y organizaciones regionales. The Chinese Government appreciates the fact that the overwhelming majority of African countries abide by the one China principle, refuse to have official relations and contacts with Taiwan and support China's great cause of reunification.El gobierno chino aprecia el hecho de que la inmensa mayoría de los países africanos respetan el principio de una sola China, se niegan a tener contactos y relaciones oficiales con Taiwán y otorgan gran apoyo a la reunificación de China. China stands ready to establish and develop state-to-state relations with countries that have not yet established diplomatic ties with China on the basis of the one China principle. China está dispuesta a establecer y desarrollar las relaciones interestatales con los países que aún no han establecido relaciones diplomáticas con China sobre la base del principio de una sola China" (Du, 2010: 2). 
nacional. Se establece que en los primeros años del nuevo siglo asistimos a una continuación de los cambios complejos y profundos en la situación internacional, a la vez que presenciamos un mayor avance de la globalización; sin embargo, la paz y el desarrollo continúan siendo los principales desafíos de nuestro tiempo. Así, salvaguardar la paz, promover el desarrollo y fortalecer la cooperación "representa la tendencia histórica irresistible". Por otro lado, se señala que "los factores de desestabilización e incertidumbre en la situación internacional están en alza"; por lo tanto, "la paz sigue siendo evasiva y de desarrollo más apremiante" (FCCA, 2006).

Al final, la conclusión es clara: sin un auténtico desarrollo, la paz siempre estará lejos de ser alcanzada. Aquí es donde entra China, recordando la visión jerárquica del mundo que en la larga tradición imperial China poseyó sobre sí misma y su destino: China, como el mayor país en desarrollo en el mundo, está dispuesta a establecer relaciones de amistad y cooperación con todos los países sobre la base de los "Cinco principios de coexistencia pacífica", "con el fin de contribuir a la paz, la estabilidad y la prosperidad común de todo el mundo" (FCCA, 2006: 1). ${ }^{17}$

En esta construcción discursiva de la realidad se mira al continente africano como una fuerza importante para la paz y el desarrollo porque abarca el mayor número de países en desarrollo. África tiene una larga historia, cuenta con un inmenso territorio, es rica en recursos naturales y posee un enorme potencial para su desarrollo. Después de largos años de lucha, los pueblos africanos se liberaron del dominio colonial, acabaron con el apartheid, ganaron su independencia y emancipación y de esta manera contribuyeron al progreso de la civilización (FCCA, 2006). Aquí se puede apreciar, entre líneas, esta doble posición del gobierno chino al invocarse la historia: China y África compartieron un pasado común de explotación colonial, y ahora China, que ha superado esa etapa de la historia y al ser el mayor país en vías de desarrollo

17. Desde el constructivismo se parte del hecho de que las identidades son construidas socialmente (Wendt, 1999). Estas identidades evidentemente tienen profundas raíces históricas. En este sentido, la visión jerárquica del sistema internacional y la responsabilidad que implica ocupar el puesto más alto en este mundo jerárquico se deriva del pensamiento clásico de Confucio, quien propuso la piedad filial como uno de los principios rectores para un buen gobierno y que se materializaba en las cinco relaciones que debían respetarse como reflejo del orden natural: gobernante-gobernado, padre-hijo, esposo-esposa, hermano mayor-hermano menor, y amigo-amigo. 
—en la figura metafórica del hermano mayor — tiene la responsabilidad de "ayudar" a los demás en ese camino de liberación y desarrollo.

Las relaciones de China con África son caracterizadas, por lo tanto, por una "profunda amistad". De esta manera se establece que desde la fundación de la República Popular, China "ha proporcionado asistencia en la medida de su capacidad a los países africanos" (FCCA, 2006). La sinceridad, la igualdad y el beneficio mutuo, sumados a la solidaridad y el desarrollo comunes, constituyen los principios rectores de los vínculos entre China y África, y la cooperación la fuerza motriz de estas relaciones.

\section{Cuadro 1}

Aspectos de cooperación entre China y África

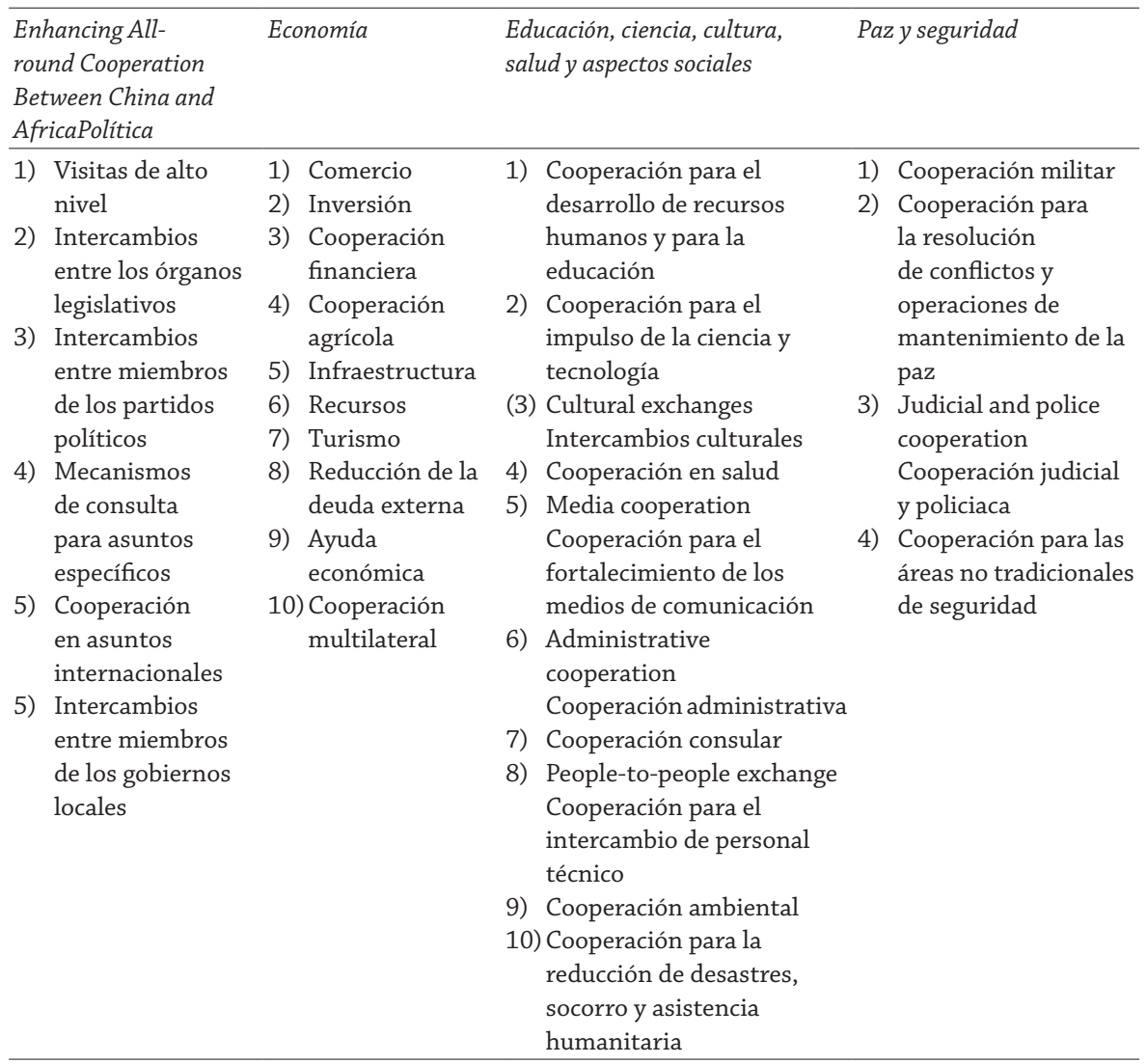

Fuente: Foro de Cooperación China-África, 2006. 


\section{China en África: el Foro sobre Cooperación China-África}

Las relaciones China-África iniciaron en el año 1956 con el establecimiento de las relaciones diplomáticas con Egipto (Brown y Chun, 2009). ${ }^{18}$ Desde entonces las posturas chinas y africanas han coincidido en numerosos foros internacionales, en los que el discurso predominante ha sido enmarcado por las ideas de beneficio mutuo basado en una política de no intervención en los asuntos internos de cada Estado. Este discurso se ha mantenido prácticamente intacto a pesar de los cambios en los intereses y prioridades de la política exterior de China en estos años.

En el primer periodo de las relaciones sinoafricanas el vínculo de China con la región fue influenciado profundamente por el aspecto ideológico del comunismo chino, que se enmarcaba en el contexto de la Guerra Fría; la élite burocrática consideraba que el rol que China debía desempeñar era el de apoyar los movimientos de liberación nacional que sacudían al continente africano (Taylor, 2010). De hecho, en la década de 1970 el número de proyectos de ayuda humanitaria que China tenía en África era similar al que Estados Unidos tenía en la región (Taylor, 2010).

Esta visión cambió radicalmente con el arribo de Deng Xiaoping al poder y las reformas económicas que se emprendieron para transformar la estructura de una economía planificada, a otra que se basara en las fuerzas del mercado. Múltiples tareas debían enfrentar los líderes chinos para generar una transición que consolidara un modelo económico distinto al que se había implementado en los primeros 30 años de la República Popular. La cooperación para el desarrollo de otras regiones del mundo no fue en ese entonces una prioridad de la política china. El apoyo a los movimientos de liberación nacional ya no tenía cabida en la nueva percepción que del mundo realizaba la élite burocrática. Este hecho se reflejó en la declinación de las relaciones sinoafricanas, en la disminución de la asistencia que China proporcionaba a la región y en la decadencia del intercambio comercial (Li, 2008). Era evidente que en la década de 1980 China había perdido totalmente el interés en África.

18. Actualmente son solamente cuatro Estados africanos los que no mantienen relaciones diplomáticas con China, ya que reconocen a Taiwán como un país independiente. Estos Estados son: Burkina Faso, Gambia, Santo Tomé y Príncipe, y Suazilandia (República de China, 2011). 
Sin embargo, la postura de China pasó de un extremo a otro después de los acontecimientos de junio de 1989 de la plaza de Tiananmen. La crisis que experimentaron las relaciones de China con los países occidentales y las duras críticas de las que fueron objeto los líderes del país, generaron un replanteamiento de la política exterior. El discurso antiimperialista y antihegemónico fue revivido enmarcándolo en las relaciones de China con los países en vías de desarrollo, particularmente los países africanos (Taylor, 2011). El discurso presentado fue que al final de la Guerra Fría el mundo era amenazado por nuevas aspiraciones hegemónicas de un solo país —evidentemente, Estados Unidos-; por lo tanto, era necesario impulsar el multilateralismo y garantizar una política de no intervencionismo. Esta postura acercó nuevamente a China con África.

De esta manera se reactivaron las relaciones entre China y los países africanos bajo una nueva perspectiva que dejó a un lado la ayuda unilateral, para centrarse en el concepto de ganar-ganar (Li, 2008). El principal detonante para el establecimiento de este nuevo marco de acción fue la visita del entonces primer ministro Jiang Zemin por África en 1996. En este viaje Jiang visitó Kenia, Namibia, Egipto, Mali, Zimbabue y Etiopía; en este último país, en el marco de la Organización de la Unión Africana, hizo un llamado a establecer una nueva relación histórica de amistad entre China y África. Esta relación, desde el punto de vista de Jiang, debía basarse en cinco principios: una sincera amistad entre China y África, trato igualitario y respetuoso de la soberanía y los asuntos internos; búsqueda del desarrollo común bajo las bases del beneficio mutuo; fortalecimiento de la cooperación en los asuntos externos e interés por crear un mundo mejor (Taylor, 2010). De esta manera se consolidó el binomio de beneficio mutuo y no injerencia en los asuntos internos como la nueva fórmula para la cooperación (Checa-Artasu, 2011).

En octubre de 1999 el primer ministro Jiang Zemin convocó a los jefes de Estados de los países africanos y al presidente de la Unión Africana a una reunión para establecer un foro que funcionara como un mecanismo permanente de contacto entre los países africanos y China. ${ }^{19}$ Un año después la reunión se llevó a cabo en Beijing con la presencia de seis jefes de Estados africanos, de 80 ministros de comercio y relaciones exteriores de 45 Estados

19. A esta reunión fueron invitados todos los Estados que mantenían relaciones diplomáticas, más los ocho países que en ese momento no mantenían relaciones con China, que fueron invitados como observadores. 
africanos, así como representantes de 17 organizaciones regionales. Los temas de la conferencia se dividieron en cuatro mesas de trabajo: comercio, reforma económica - con énfasis en el modelo chino-, erradicación de la pobreza y desarrollo sustentable, y cooperación en el campo de la educación, la salud y la ciencia y la tecnología (FCCA, 2000).

Los principales compromisos del primer foro sobre la cooperación ChinaÁfrica fueron:

- Incrementar la ayuda para el desarrollo, específicamente en la promoción de las industrias africanas, con la obtención y uso de materiales locales y creando fuentes de trabajo para los africanos.

- Formar el Consejo de Negocios China-África.

- Establecer un centro de exhibición de productos africanos en China.

- Proveer asistencia a África a través de la promoción del desarrollo económico y social en el marco de una cooperación Sur-Sur.

- Fomentar la inversión para el desarrollo de la infraestructura turística.

- Proveer fondos especiales para el establecimiento de alianzas entre corporaciones chinas y africanas para proyectos específicos.

- Impulsar a las corporaciones chinas para contribuir en la construcción de infraestructura de los países africanos.

- Establecer el Fondo para el Desarrollo de Recursos Humanos Africanos para la capacitación de personal africano (Taylor, 2010).

El primer Foro sobre la Cooperación China-África puede ser considerado exitoso tanto por los acuerdos alcanzados como por la proyección obtenida para China. En primer lugar, los dos documentos generados en el Foro - la Declaración de Beijing sobre el Foro China-África y el Programa para la Cooperación Económica y Social China-África - fueron fundamentales para establecer los parámetros de la relación entre ambas partes. En segundo lugar, el foro estableció un mecanismo para el futuro diálogo entre China y África. En tercer lugar, el foro reflejó el deseo de las élites chinas y africanas para promover la posición del Sur en los foros internacionales y plantear la necesidad de reconfigurar la política internacional en beneficio de los países en desarrollo. Finalmente, el Foro fue un espacio para concretizar un proyecto tangible sobre la cooperación Sur-Sur (Taylor, 2010).

La segunda conferencia Ministerial del Foro sobre Cooperación ChinaÁfrica se realizó en la ciudad de Addis Abba en diciembre de 2003. A esta reunión asistieron el primer ministro chino, Wen Jiabao; el primer ministro 
etíope, Meles Zenawi; seis presidentes africanos, tres vicepresidentes y dos primeros ministros, así como Alpha Oumar Konaré, presidente de la Comisión de la Unión Africana. Asimismo participaron más de 70 ministros de China y 44 de países africanos, responsables de asuntos exteriores y cooperación económica internacional, y representantes de algunas organizaciones regionales e internacionales africanos asistieron a la conferencia (FCCA, 2003a).

En el Plan de Acción de Addis Abeba para el FCCA 2004-2006, el Gobierno chino estableció los siguientes compromisos:

- China aumentaría los recursos asistenciales a los países africanos en el marco de FOCAC durante los siguientes tres años.

- China apoyaría la cooperación para la formación de recursos humanos hasta para 10 mil técnicos en diferentes campos del conocimiento.

- China abriría el mercado y otorgaría acceso libre de aranceles al mercado chino a algunos productos de los países menos adelantados de África.

- China ampliaría la cooperación turística con África y daría a ocho países africanos (Etiopía, Kenia, Tanzania, Zambia, Mauricio, Seychelles, Zimbabue y Túnez) el estatus de destino aprobado para la salida de turistas chinos.

- China patrocinaría el "Encuentro en Beijing" - un festival de arte internacional centrado en las artes de África-y el "Viaje de la cultura china a África”, el cual difundiría la riqueza de la civilización china en África.

- China incrementaría el número de intercambios de personas con África y llevaría a cabo el Festival de la Juventud China-África que se celebró en China en el año 2004 (FCCA, 2003b).

Los días 4 y 5 de noviembre de 2006 se llevó a cabo la tercera conferencia ministerial del Foro sobre Cooperación China-África en la ciudad de Beijing. En esa ocasión se contó con la presencia de 24 jefes de Estado y ministros de 48 países africanos, 1,700 delegados y más de 20 organizaciones de las Naciones Unidas y organizaciones regionales africanas. Se calcula que más de mil reporteros, entre ellos 300 de países africanos, dieron seguimiento a la reunión (Taylor, 2010).

Como resultado de la reunión se elaboró la Declaración de Beijing, en la que se estableció que el Foro sobre Cooperación China-África se había convertido en una importante plataforma para el diálogo colectivo y un mecanismo eficaz para la cooperación pragmática; por lo tanto, los miembros participantes acordaron aumentar el papel del Foro con la intención de fortalecer la coo- 
peración entre ambas partes y presentar de manera conjunta, ante los foros internacionales, aquellas posturas que apoyaran el desarrollo de los países con menos oportunidades (FCCA, 2006a). Asimismo, se proclamaba un nuevo tipo de asociación estratégica entre China y África caracterizada por "la igualdad política y la confianza mutua" (FCCA, 2006a: 1).

Entre las medidas a las que se comprometieron los asistentes al III Foro Ministerial, destacaban:

- El incremento de visitas de alto nivel, la realización de diálogos estratégicos, el aumento de la confianza política mutua y la promoción de una amistad duradera.

- La profundización y ampliación de la cooperación, el fomento al comercio bilateral y a las inversiones, la exploración de nuevas formas de cooperación dando máxima prioridad a la cooperación en agricultura, infraestructura, industria, pesca, informática, salud pública y la capacitación del personal.

- El aumento del diálogo entre diferentes culturas, promoviendo a las personas a través de intercambios y la interacción, particularmente entre los jóvenes; el impulso a los intercambios y la cooperación en ámbitos como la cultura, la ciencia y la tecnología, la educación, el deporte, el medio ambiente, el turismo y los asuntos de la mujer.

- El incremento en la cooperación internacional para abordar conjuntamente las amenazas globales de seguridad y los desafíos no tradicionales a la seguridad (FCCA, 2006a).

En esa ocasión el gobierno chino aprovechó para lanzar la Nueva Alianza para el Desarrollo de África con la intención de activar la participación de empresas chinas en el mercado africano. Este plan se concentraba en ciertos países africanos que por sus recursos naturales, su crecimiento económico o sus fuertes vínculos diplomáticos con China representaban una excelente oportunidad de negocios. De esta manera, el plan chino contemplaba facilitar tres mil millones de dólares en préstamos y dos mil millones en créditos preferentes, además de establecer un fondo de cinco mil millones de dólares para impulsar las inversiones de empresas chinas, así como cancelar $10 \mathrm{mil}$ millones de dólares de la deuda de los países más pobres del conteniente africano (Checa-artasu, 2011). El plan de acción derivado de esta reunión constituyó un programa muy amplio que contemplaba aspectos políticos, económicos, sociales y culturales, y abarcaba temas desde visitas de alto nivel 
hasta la cooperación para asuntos no tradicionales en materia de seguridad pública (FCCA, 2006b).

Entre los puntos más importantes del plan de acción destaca el compromiso del gobierno chino de duplicar los fondos asistenciales entre los años 2006 y 2009; abrir el mercado chino de 190 a 440 productos de importación, con una tasa preferencial de $0 \%$ en el pago de aranceles, a los países menos desarrollados que mantuvieran vínculos diplomáticos con China; establecer de tres a cinco zonas de comercio y cooperación económica en África para los siguientes tres años; capacitar a 15 mil profesionistas africanos; enviar a 100 técnicos expertos en agricultura; construir 30 hospitales y crear un fondo de 300 millones de yuanes para prevenir la malaria; construir 100 escuelas rulares y otorgar cuatro mil becas gubernamentales para estudiantes en el año 2009 (FCCA, 2006b).

La cuarta conferencia ministerial del Foro sobre Cooperación China-África se llevó a cabo en la ciudad egipcia Sharm El Sheik en noviembre de 2009. La conferencia contó con la presencia de 17 jefes de Estado de los países africanos y del primer ministro chino Wen Jiabao (FCCA, 2009a). En comparación con la conferencia de Beijing de tres años antes, esta reunión fue más mesurada, a la que asistieron solamente los ministros de economía y relaciones exteriores de los países convocados y en la que no participó el presidente de China $\mathrm{Hu}$ Jintao (Taylor, 2010).

En la reunión el Gobierno chino asumió ocho compromisos para dar continuidad a la política de cooperación iniciada nueve años atrás. Entre estos compromisos, el primero de ellos se enfocaba en combatir el cambio climático. En este sentido, China se comprometía a facilitar la cooperación para el monitoreo satelital del clima, fortalecer la utilización de nuevas fuentes energéticas, prevenir y controlar la desertificación e impulsar la protección ambiental de las ciudades. Como parte de este compromiso, China garantizó la construcción de 100 proyectos energéticos que incluían la difusión de tecnología basada en el aprovechamiento de energía solar, el uso del biogás y el desarrollo de centrales microhidroeléctricas (FCCA, 2009b).

Entre otras medidas, el Gobierno chino asumió profundizar la cooperación científica y tecnológica; aportar 10 mil millones de dólares para fortalecer la capacidad financiera de algunas naciones africanas; continuar con la aplicación de tarifas preferenciales para la importación de productos africanos de los países menos desarrollados; incrementar el número de centros de investigación y desarrollo en el campo de la agricultura; profundizar la cooperación en el 
campo de la medicina y la salud a través de la donación de equipo médico, la construcción de 30 hospitales y 30 centros de prevención y tratamiento de la malaria; ampliar la cooperación para la educación y la formación de recursos humanos por medio de capacitar a 1,500 directores y profesores, construir 50 escuelas y otorgar hasta 5,500 becas gubernamentales; finalmente, multiplicar los intercambios culturales para favorecer una mejor comprensión de China y África y de las medidas más acertadas para el desarrollo (FCCA, 2009b).

La cuarta conferencia ministerial consolidó el Foro sobre la Cooperación China-África como un mecanismo del más alto nivel desde el cual China ha proyectado su propia visión sobre lo que el mundo debe ser, sobre el papel que la cooperación en este nuevo mundo puede desempeñar para establecer un orden internacional distinto al actual y sobre el liderazgo que China quiere tener al respecto. Si bien es cierto que las conferencias ministeriales son un espacio privilegiado para, desde la palabra, erigir o fortalecer las identidades que son construidas socialmente y que proyectan ciertas imágenes de los Estados a partir de sus élites burocráticas, al mismo tiempo han sido un lugar desde el cual se muestran o se comprometen acciones concretas emprendidas en el campo de la cooperación que al final de cuentas impactan materialmente en la vida de las personas, sus comunidades y los Estados en que tienen lugar. En otras palabras, son las ideas identitarias generadas desde el discurso y las acciones materiales que las acompañan, las que conjuntamente moldean el escenario internacional y fortalecen o debilitan la imagen internacional de los actores estatales en este escenario y su capacidad de acción.

\section{Consideraciones finales}

El mundo ha cambiado significativamente en los últimos 20 años. El escenario internacional pasó de ser un espacio dividido en dos grandes bloques que se ordenaba y se entendía desde la dinámica de un mundo bipolar, a un lugar de incertidumbres y desesperanzas, en las que la hegemonía de un solo país o un bloque de países no parece estar garantizada, ni el orden de lo que comúnmente se ha denominado el nuevo sistema internacional parece claro. Al mismo tiempo han surgido nuevos desafíos que rebasan en no pocas ocasiones el margen de acción de los Estados-nación y nuevas amenazas a la seguridad de los Estados están presentes todos los días, haciendo que en cierta medida el mundo de hoy sea más incierto que el que se conocía hace una generación. 
En este contexto aparecen nuevos actores que reclaman una mayor participación en los asuntos internacionales, apelando al tamaño de su economía o al peso de su población. Entre estos actores se encuentra China como una potencia emergente que reclama un espacio de acción en el escenario internacional conforme a sus propios intereses. Estos intereses, no hay que olvidarlo, se originan no solamente de las necesidades materiales que condicionan las demandas de China y su sociedad, sino también son constituidos a partir de las identidades construidas por la élite burocrática, enmarcada en una tradición histórica y un contexto social particular, moldeado por la imagen de la nueva China que se construye y se comunica todos los días.

La presencia de estos países que reclaman un orden mundial diferente, en sí mismo no representa el surgimiento de un escenario internacional cualitativamente distinto. La conformación de un orden mundial alterno al modelo actual es un hecho que aún no ha sido consumado. Sin embargo, lo que sí se puede observar, en el caso chino, es la existencia de una estrategia mediática por parte del Partido Comunista para fortalecer la imagen de China como país protagónico en la arena internacional que puede contribuir a la conformación de un escenario distinto. Bajo la lógica de que China se relaciona de manera diferente con los demás países y que sus intereses y motivaciones son distintos, se está gestando una identidad del Estado chino conforme a sus aspiraciones mundiales. Esta identidad, claro está, se comunica una y otra vez no sólo al interior del Estado chino, sino también a los demás miembros de la comunidad internacional.

De esta manera, el Foro sobre Cooperación China-África es un espacio en el que se concretan las nuevas relaciones que tienen lugar a partir de las nuevas identidades construidas y comunicadas socialmente. Si bien es cierto que este trabajo muestra únicamente el aspecto discursivo del Foro China-África, es claro que la relación de China con los países africanos es variada, profunda y compleja, y no solamente se puede explicar desde el discurso. No obstante, es necesario tener presente que este discurso ha rebasado el mundo de las ideas, para materializarse en un mecanismo institucional, regular y definido, en el que se enmarcan las relaciones de China con África.

Así, más allá de las medidas efectivas o limitadas que el Foro sobre Cooperación China-África ha representado para las partes involucradas, es un hecho que actualmente se trata de un mecanismo consolidado con más de 10 años de existencia, que ha permitido la reunión de alto nivel entre funcionarios chinos y africanos. Un estudio diferente que se centre en el análisis 
detallado de las estructuras materiales que enmarcan también la relación de China con África permitiría conocer qué tan coherente es la visión discursiva con la realidad. ¿Es el foro de cooperación propaganda pura y simple? ¿O es una visión ideacional que tiene su impacto en las acciones del Estado chino?

Al mismo tiempo, el Foro sobre Cooperación China-África ha significado un modelo de cooperación Sur-Sur orientado en una nueva dimensión, en el que se resalta que la cooperación puede tener lugar sin ser condicionada por los asuntos internos de cada Estado. Este modelo, en sí mismo, resulta más que atractivo para ciertos países, que escapan así de una cooperación condicionada por su política interior que está al margen de las pautas deseables desde el liberalismo político. La fórmula china, expresada en el binomio cooperación sin intervención política, es una voz melodiosa que cautiva a más de un país que tiene problemas para la rendición de cuentas transparentes o de respeto a los derechos humanos.

Finalmente, este foro es, además, un espacio para la proyección de la nueva imagen de China, acompañado de un discurso en particular que replantea la reconfiguración del escenario internacional hacia un escenario caracterizado por el multilateralismo. Multilateralismo que, desde la perspectiva de China, no significa igualdad, sino más bien un mundo ordenado por la jerarquía, donde China pretende ocupar el lugar del hermano mayor: guía, impositor y modelo a seguir. ng

\section{Referencias bibliográficas}

Addler, Emmanuel (1997), "Seizing the Middle Ground: Constructivism in World Politics", European Journal of International Relations, 3(3), pp. 319-363.

Anguiano, Eugenio (2008), "China como potencia mundial: Presente y futuro", en Cornejo, Romer (ed.), China, radiografía de una potencia en ascenso, México, D. F.: El Colegio de México, p. 19-120.

Banco Mundial (2001), Datos de países: China. Recuperado de: http://datos. bancomundial.org/pais/china

Brown, Kerry, y Chun, Zhang (2009), China in Africa: Preparing for the Next Forum for China Africa Cooperation, Londres: Chatham House.

Checa-Artasu, Martín (2011), "La República Popular de China en la África subsahariana. Notas de una relación geoeconómica contemporánea”, México y la Cuenca del Pacífico, 14(40), pp. 43-73. 
China Radio International (2005), Coexistencia pacífica: Solicitud presentada por la historia. Recuperado de: http://espanol.cri.cn/141/2005/04/20/1@58707. htm

Du, Xiaconcog (2010), China's role in Africa. Recuperado de: http://www.focac. org/eng/zfgx/dfzc/t689653.htm

Faust, John, y Kornberg, Judith (1995), China in World Politics, Londres: Lynne Rienner Publishers.

Foro sobre la Cooperación China-África (2000), The First Ministeral Conference of FOCAC. Recuperado de: http://www.focac.org/eng/ltda/dyjbzjhy/ CI12009/t157577.htm

(2003a), The Second Ministeral Conference of FOCAC. Recuperado de: http://www.focac.org/eng/ltda/dejbzjhy/CI22009/t157581.htm

-_ (2003b), Major Commitments of the Chinese side on FOCAC. Recuperado de: http://www.focac.org/eng/ltda/dejbzjhy/CI22009/t157585.htm

- - (2006a), Declaration of the Beijing Summit of the Forum on China-Africa Cooperation. Recuperado de: http://www.focac.org/eng/ltda/dscbzjhy/ DOC32009/t606841.htm

- - (2006b), Forum on China-Africa cooperation Beijing action plan (20072009). Recuperado de: http://www.focac.org/eng/ltda/dscbzjhy/ DOC32009/t280369.htm

- - (2009a), Declaration of Sharm El Sheikho of the Forum on China-Africa Cooperation. Recuperado de: http://www.focac.org/eng/ltda/dsjbzjhy/ hywj/t626388.htm

- - (2009b), Forum on China-Africa cooperation Sharm El Sheikho action plan (2007-2009). Recuperado de: http://www.focac.org/eng/ltda/dsjbzjhy/ hywj/t626387.htm

Gungwu, Wan (2008), "China and the International Order: Some historical perspectives", en Yongnian, Zheng, y Gungwu, Wan (eds.), China and the new international order. Abindong, Oxon: Routledge, pp. 21-31.

Guzzini, Stefano (2000), "A Reconstruction of Constructivism in International Relations", European Journal of International Relations, 6(2), pp. 147-182. Halper, Stefan (2010), The Beijing Consensus: How China's Authoritarian Model Will Dominate the Twenty-First Century. Nueva York: Basic Books.

Hu, Jintao (2011), Full text of Chinese President Hu Jintao's remarks at BRICS Leaders Meeting: Broad Vision, Shared Prosperity. Recuperado de: http:// www.gov.cn/english/2011-04/14/content_1844180.htm 
Jacques, Martin (2009), When China Rules the World: The end of the western world and the birth of a new global order. Nueva York: Penguin Press.

Lemus, Daniel (2010), "La construcción de la nueva imagen china a través de los medios: Análisis de la ceremonia de inauguración de los Juegos Olímpicos de Beijing y el desfile del 60 aniversario de la República Popular China", Confines, de Relaciones Internacionales y Ciencia Política, 6(2), pp. 51-76.

Leonard, Mark (2008), ¿Qué piensa China? Barcelona: Icaria Antrazyt.

Li, Anshan (2008), "China's New Policy toward Africa”, en Rotberg, Robert I. (ed.), China into Afirca: Trade, Aid and Influence, Cambridge: World Peace Foundation, pp. 21-49.

Li, Zhaoxing (2005), La paz, el desarrollo y la cooperación: Bandera de la diplomacia china en la nueva época. Recuperado de: http://www.fmprc.gov.cn/ esp/wjdt/wjzc/t208342.htm

Lora, Eduardo (2007), ¿Debe América Latina temer a China?, Nueva York: Banco Interamericano de Desarrollo.

Ministerio de Asuntos Exteriores (2003), Posición china para la cooperación SurSur. Recuperado de: http://www.fmprc.gov.cn/esp/wjdt/wjzc/t25296.htm Oficina de Información del Consejo de Estado (2011), El desarrollo pacífico de China. Recuperado de: http://www.chinadaily.com.cn/china/2011-09/06/ content_13630926.htm

Republic of China (2011), The Republic of China Yearbook 2010, Taipei: Government Information Office. Recuperado de: http://www.gio.gov.tw/ taiwan-website/5-gp/yearbook/

Reus-Smit, Christian (2009), "La estructura constitucional de la sociedad internacional y la naturaleza de las instituciones fundamentales", en Santa Cruz, Arturo (ed.), El constructivismo y las relaciones internacionales, México, D. F.: CIDE, pp. 175-221.

Rotberg, Robert I. (2008), "China's Quest for Resources, Oportunities and Influence in Africa", en Rotberg, Robert I. (ed.), China into Afirca: Trade, Aid and Influence, Cambridge: World Peace Foundation, pp. 1-20.

Rupp, Stephanie (2008), "Africa and China: Engaging Postcolonial Interdependences", en Rotberg, Robert I. (ed.), China into Africa: Trade, Aid and Influence, Cambridge: World Peace Foundation, pp. 65-86.

Santa Cruz, Arturo (2009), "Introducción", en Santa Cruz, Arturo (ed.), El constructivismo y las relaciones internacionales, México, D. F.: CIDE, pp. 9-37. Secretaría de Relaciones Exteriores (2011), Cooperación Internacional de México. Recuperado de: http://dgctc.sre.gob.mx/html/coop_int_mex.html 
Taylor, Ian (2010), China's New Role in Africa, Londres: Lynne Rieener Publisher.

(2011), The Forum on China-Africa Cooperation (FOCAC), Oxon: Routledge.

Wendt, Alexander (1999), Social Theory of International Politics, Cambridge: Cambridge University Press.

Xinhua (2010), Agencia de noticias Xinhua lanza servicio mundial de televisión en inglés. Recuperado de: http://spanish.news.cn/principales/201004/30/c_13273781.htm

Zalewski, Marysia, y Enloe, Cynthia (1995), “Questions about identity”, en Booth, Ken, y Smith, Steve (eds.), International Relations Theory Today, Pennsylvania: Pennsylvania University Press, pp. 279-305.

Zhefuss, Maja (2002), Constructivism in International Relations: The Politics of Reality, Cambridge: Cambridge University Press. 


\section{Crecimiento económico y desarrollo financiero en Asia-Pacífico}

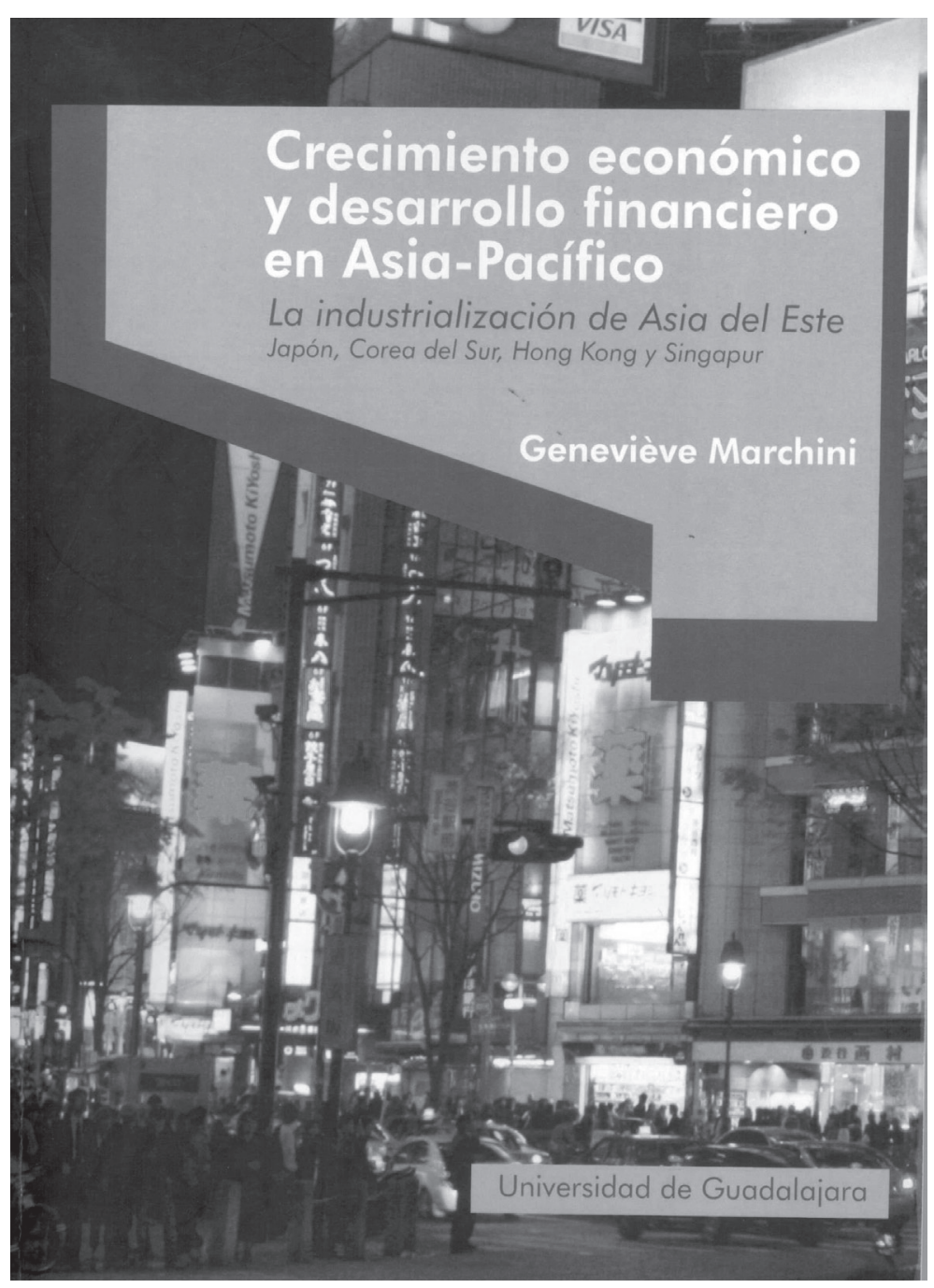

